\title{
Iterative methods for Lyapunov vectors and singular vectors and atmospheric predictability
}

\author{
Mozheng Wei* Jorgen S. Frederiksen*
}

(Received 7 August 2000)

\begin{abstract}
Iterative methods are used to generate Lyapunov vectors (LVs) and singular vectors (svs). Their roles in describing atmospheric error growth and predictability are studied. LVs are produced by evolving a set of initially random perturbations and using a modified GramSchmidt re-orthogonalisation to ensure their orthogonality. The structures of LVs and SVs, and finite-time normal modes (FTNMs), are com-
\end{abstract}

*Meteorology CRC, CSIRO Atmospheric Research, Australia.

mailto:mwei@splash.princeton.edu and mailto: jorgen.frederikson@dar.csiro.au

${ }^{0}$ See http: //anziamj . austms .org. au/V42/CTAC99/Wei2 for this article and ancillary services, (C) Austral. Mathematical Soc. 2000. Published 27 Nov 2000. 
pared with patterns of 100 errors evolving in a barotropic tangent linear model.

\section{Contents}

2 Singular Vectors and Lyapunov Vectors C1503 2.1 Model description . . . . . . . . . . . . . . . . . C1504 2.2 Singular vectors . . . . . . . . . . . . . . . . . C1505 2.3 Lyapunov vectors . . . . . . . . . . . . . C1506

3 Properties of LVs, SVs and FTNMs

C1509

4 Statistics of Error Growth

$\mathrm{C} 1514$

5 Conclusions

C1517

References

C1518 


\section{Introduction}

We use iterative methods to generate Lyapunov vectors (LVs) and singular vectors (SVs) and examine their roles in atmospheric error growth and predictability. These perturbations are studied using the barotropic tangent linear model with time dependent basic states taken from observations. LVs are obtained by evolving a set of initially random perturbations and using a modified Gram-Schmidt re-orthogonalisation to ensure the orthogonality of the LVs. We discuss the relative merits of generating SVs using Arnoldi or Lanczos methods compared with explicit construction of the propagator matrix and singular value decomposition. The structures of LVs and SVs, and finite-time normal modes (FTNMs), are compared with patterns of evolved random errors. Our aim is to determine the type of perturbations which can efficiently represent analysis errors and that are likely to be useful for constructing initial ensembles for ensemble weather forecasts $[4,5,2]$.

Section 2 describes iterative methods for constructing LVs and SVs while in Section 3 we study the structures and amplification of LVs, SVs and FTNMs during atmospheric blocking. In Section 4 we present pattern correlations between evolved random errors and dynamical vectors and our conclusions are summarised in Section 5.

\section{Singular Vectors and Lyapunov Vectors}




\subsection{Model description}

We use the spectral barotropic tangent linear model, described in detail in Section 2 of [7] for studying LVs and svs and error growth. With $\mathrm{x}$ denoting the column vector of real and imaginary parts of spectral coefficients of perturbation fields, the tangent linear spectral equations can be written in the form

$$
\frac{d \mathbf{x}(t)}{d t}=\mathbf{M}(t) \mathbf{x}(t)
$$

where $\mathbf{M}(t)$ is the tangent linear operator evaluated on the observed trajectory $\mathbf{X}(t)$. The formal solution of equation (1) is

$$
\mathbf{x}(t)=\mathbf{G}\left(t, t_{0}\right) \mathbf{x}\left(t_{0}\right),
$$

where $\mathbf{G}\left(t, t_{0}\right)$ is called the propagator. As described in [7] our horizontal resolution is specified by a rhomboidal truncation wave number $R=15$, so that spectral components have zonal wave number $|m| \leq 15$, and total wave number $l \leq|m|+15$. We use a half-hour time step to solve our equations using a leap frog scheme and the results are for both the inviscid case and a case with a typical magnitude of the viscosity. Comparison of the respective results gives an indication of the sensitivity to dissipation. In the viscous case, we use a combination of a linear drag $(\eta \zeta)$ and a $\nabla^{4}$ diffusion $\left(\eta^{\prime} \nabla^{4} \zeta\right)$, where $\zeta$ is the vorticity; the coefficients of viscosity are chosen as $\eta=8.4 \times 10^{-7} \mathrm{~s}^{-1}$ and $\eta^{\prime}=2.5 \times 10^{16} \mathrm{~m}^{4} \mathrm{~s}^{-1}$. We use $300-\mathrm{mb}$ streamfunction fields taken from daily observations and linearly interpolated to obtain the time-dependent basic states needed every half-hour. 


\subsection{Singular vectors}

Singular vectors are norm dependent structures which are used at some weather prediction centres as perturbations in ensemble forecasts $[4,2]$. For the Euclidean inner product $\langle\mathbf{x} ; \mathbf{y}\rangle$, the norm of the perturbation at time $t$ is given by

$$
\|\mathbf{x}(t)\|^{2}=\langle\mathbf{x}(t) ; \mathbf{x}(t)\rangle=\left\langle\mathbf{G}^{\dagger}\left(t, t_{0}\right) \mathbf{G}\left(t, t_{0}\right) \mathbf{x}\left(t_{0}\right) ; \mathbf{x}\left(t_{0}\right)\right\rangle,
$$

where $\mathbf{G}^{\dagger}\left(t, t_{0}\right)$ denotes adjoint operator or Hermitian conjugate matrix with respect to the Euclidean inner product. The operator $\mathbf{G}^{\dagger}\left(t, t_{0}\right) \mathbf{G}\left(t, t_{0}\right)$ is symmetric and has right eigenvectors $\mathbf{v}^{\nu}(\nu=1,2, \ldots, n)$ and left eigenvectors $\mathbf{u}^{\nu}$ each of which form a complete orthonormal basis set. The singular vectors of $\mathbf{G}\left(t, t_{0}\right)$ satisfy the equations

$$
\mathbf{G}^{\dagger}\left(t, t_{0}\right) \mathbf{G}\left(t, t_{0}\right) \mathbf{v}^{\nu}=\left(\sigma^{\nu}\right)^{2} \mathbf{v}^{\nu} ; \quad \mathbf{G}\left(t, t_{0}\right) \mathbf{G}^{\dagger}\left(t, t_{0}\right) \mathbf{u}^{\nu}=\left(\sigma^{\nu}\right)^{2} \mathbf{u}^{\nu} .
$$

where the real eigenvalues $\sigma^{\nu} \geq 0$. The $\sigma^{\nu}$ are the singular values of $\mathbf{G}\left(t, t_{0}\right)$. The singular vectors and values can be calculated by iterative solvers based on the Arnoldi method [3, 6] or Lanczos methods [4]. The action of $\mathbf{G}^{\dagger}\left(t, t_{0}\right) \mathbf{G}\left(t, t_{0}\right)$ is equivalent to integrating the linearised equations (1) or (2) from time $t_{0}$ to $t$, and followed by integrating the corresponding adjoint equations backward from $t$ to $t_{0}$. The dominant singular values and vectors can be approximated by repeating this process. Both Arnoldi and Lanczos methods are variants of Krylov subspace methods and while they are efficient 
for calculating Svs for large systems of differential equations they need corresponding adjoint equations which may not be readily available. Since the operator $\mathbf{G}^{\dagger}\left(t, t_{0}\right) \mathbf{G}\left(t, t_{0}\right)$ is symmetric, the Lanczos method is more efficient.

When the dimension of $\mathbf{G}\left(t, t_{0}\right)$ is not too large, an alternative strategy, which does not require the formulation of adjoint equations, is to calculate the matrix form of propagator and solve eqs. (4). The propagator may be constructed by integrating forward in the tangent linear model $n$ unit vectors $\mathbf{x}^{i}\left(t_{0}\right)=\mathbf{e}^{i}$ where $\mathbf{e}_{j}^{i}=\delta_{i j}$ with $\delta$ the Kronecker delta function. The propagator then is

$$
\mathbf{G}\left(t, t_{0}\right)=\left(\mathbf{x}^{1}\left(t, t_{0}\right), \mathbf{x}^{2}\left(t, t_{0}\right), \ldots, \mathbf{x}^{n}\left(t, t_{0}\right)\right) .
$$

If $\mathbf{x}$ in Equation (1) consists of spectral coefficients of vorticity then the norm squared (3) is the enstrophy (EN). Similarly streamfunction spectral coefficients give streamfunction (SF) (squared) norm and velocity components give the kinetic energy ( $\mathrm{KE}$ ) norm. We shall need to study SVs in each of these norms and we refer to them as EN SVs, SF SVs and KE SVs. We shall also need to calculated the eigenvectors of the propagator which are the FTNMs of [7]; these are obtained using Arnoldi methods as described there.

\subsection{Lyapunov vectors}

A commonly used method of calculating LVs is to compute the left SVs $\mathbf{u}^{\nu}$ for a large time interval $t-t_{0} ; \mathbf{u}^{\nu}$ will converge to LVs when $t-t_{0} \rightarrow \infty$. Here we 
use an iterative method which consists of evolving a set of initial orthonormal vectors $\mathbf{p}^{\nu}\left(t_{0}\right)(\nu=1,2, \ldots, k$ and $k \leq n)$, chosen at random in the tangent space $T^{n}\left(t_{0}\right)$ at $\mathbf{X}\left(t_{0}\right)$ by integrating the perturbation equation (1) along the observed basic state flow. This method has been shown to be cheaper and easier to implement. To save computing time, $k$ can be chosen very small. Here we choose $k=n$ and all the LVs are calculated in this case. This kind of method has been used for calculating global Lyapunov exponents (LEs) [1].

Since $\mathbf{p}^{1}$ has the largest growth rate, all the perturbations will ultimately tend towards the most unstable direction associated with the largest LE. To prevent this from happening, it is necessary to perform a GramSchmidt orthogonalisation from time to time. In practice, we always carry out a modified Gram-Schmidt re-orthogonalisation which involves a double re-orthogonalisation at each time step. This ensures that at any time $t$ the vectors $\mathbf{p}^{\nu}(t)$ form an orthonormal set of vectors evolving in the tangent space.

After an initial transient period, we have a set of orthonormal vectors $\mathbf{p}^{\nu}(t)$ that characterise the local directions of stretching and contraction of perturbations. We call this set of orthonormal vectors $\mathbf{p}^{\nu}(t)$ Lyapunov vectors (LVs) at time $t$. In this paper, the instantaneous and finite-time growth rates of the LVs are called local Lyapunov exponents and finite-time Lyapunov exponents respectively. The finite-time Lyapunov exponents can be calculated as $L^{\nu}\left(t_{2}, t_{1}\right)=\frac{1}{t_{2}-t_{1}} \ln \frac{\left\|\mathbf{p}^{\nu}\left(t_{2}\right)\right\|}{\left\|\mathbf{p}^{\nu}\left(t_{1}\right)\right\|}$, where $t_{2}-t_{1}=J \Delta t(\mathrm{~J}$ is a positive integer, $\Delta t$ is a small integration time step). Clearly $L^{\nu}\left(t_{2}, t_{1}\right)$ depend on $t_{1}, t_{2}$ and position on the trajectory $\mathbf{X}(t)$; they measure the average perturbation growth 
over a given interval $t_{2}-t_{1}$. The global LEs are recovered by taking the limit $t_{2} \rightarrow \infty$. When $J=1$, we have the local Lyapunov exponents which are denoted by $L^{\nu}(t)$. Thus

$$
L^{\nu}(t)=\frac{1}{\Delta t} \ln \frac{\left\|\mathbf{p}^{\nu}(t+\Delta t)\right\|}{\left\|\mathbf{p}^{\nu}(t)\right\|} .
$$

Both $\mathbf{p}^{\nu}(t)$ and $L^{\nu}(t)$ are local properties of the dynamical system. For meteorological and climate applications, the local and finite-time growth rates are usually more relevant than global exponents. Global Lyapunov exponents only measure the long-time average exponential growth rates along different directions in the phase space. In theory, LVs at time $t$ should be calculated by starting integrations of both nonlinear and linearised equations from $-\infty$. LVs are the unique local properties of dynamical system and any unrestricted perturbations will eventually converge to the first LV (LV 1) $[5,2]$. The long-time average growth rates are given by corresponding global LEs, which are ordered from largest to smallest, so $L^{1} \geq L^{2} \geq \cdots \geq L^{n}$, and their local growth rates are described respectively by the local LES $L^{1}(t), L^{2}(t), \ldots, L^{n}(t)$. However, at any time $t$, the local growth rate of $L V 1$ is not always largest, although its long-time average growth rate $\left(L^{1}\right)$ is the largest. 


\section{Properties of LVs, SVs and FTNMs}

Here we compare the properties and evolutions of SVs and LVs during the period between 20 to 26 April 1989. During this period, blocking highlow dipoles formed at the longitudes of eastern Australia $\left(150^{\circ} \mathrm{E}\right)$ and in the central Pacific $\left(120^{\circ} \mathrm{W}\right)$. By 24 April the eastern Australian block had amplified and caused a split in the jet stream while by 26 April the central Pacific block had developed. We calculate KE SVs, SF SVs and EN SVs for this period. The results shown here are for the inviscid case. The results for the viscous case are very similar.

The SVs in each of the three norms for the optimisation period between 20 and $21, \ldots, 25$ to 26 April have been calculated (not shown). In contrast to the sensitive norm dependence of the initial SVs, the evolved SVs on 26 April are much more similar in their structures. By 26 April, all the evolved svs 1 have dipole or multipole structures, of similar scales to the central Pacific block, which are focussed in the blocking region near $120^{\circ} \mathrm{W}$. In fact, similar evolved SV structures are obtained for shorter optimisation times ending on 26 April. These structures are also quite similar to the structure of the first finite-time normal mode (FTNM) for the period from 20 to 26 April shown in Figure 1d. FTNMs are the eigenvectors of the propagator [2]. The evolved KE SVs 1 for 23-26 April and 20-26 April are displayed in Figures 1a and b respectively. Note that all the vectors shown in Figure 1 are scaled to have similar amplitudes for comparison. 


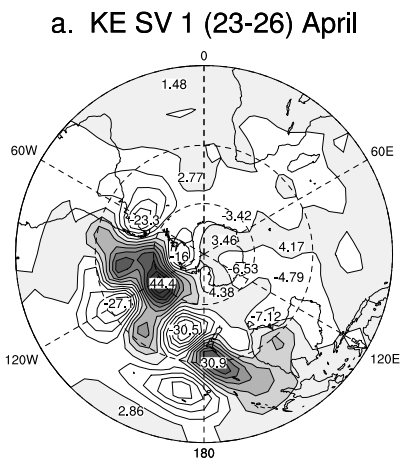

b. KE SV 1 (20-26) April

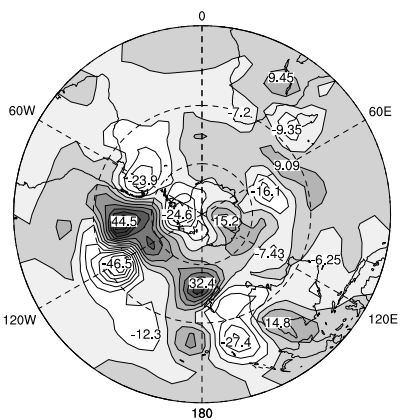

\section{c. LV 1,26 April}

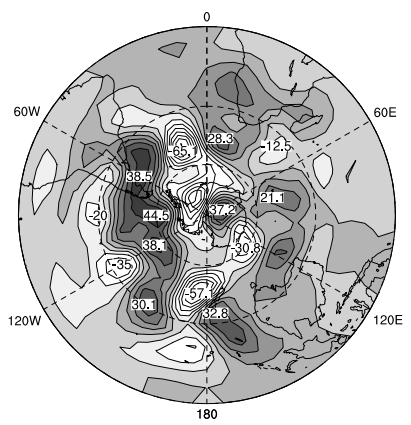

d. FTNM 1 (20-26) April

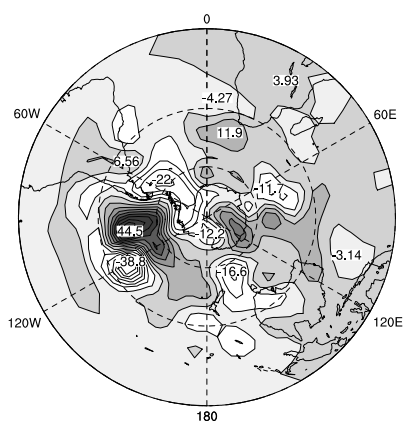

FigurE 1: The streamfunctions of dominant dynamical vectors on 26 April 1989 in the inviscid case. Shown are (a) the evolved KE SV 1 for 23 to 26 April, (b) the evolved KE SV 1 for 20 to 26 April, (c) LV 1 on 26 April and (d) FTNM 1 for 20 to 26 April 1989. 
Next, we examine the structures of dominant LVs in late April. As an example, Figure 1c shows the structures of the leading LV on 26 April. The leading LV is a local property of the system, it depends on time, and is associated with the largest global Lyapunov exponent. That is, over a sufficiently long period of time it will have the largest average growth rate of all the Lyapunov vectors. These Lyapunov vectors have been obtained by starting with random initial perturbations on 26 March 1989, integrating the tangent linear model forward and using the iterative method to obtain the LVs and global Lyapunov exponents. In principle, the integration should start from $-\infty$, but in practice a month is sufficient time.

LV 1 on 26 April has significant amplitude in the blocking region between $60^{\circ} \mathrm{W}$ and $120^{\circ} \mathrm{W}$; however, it also has a number of significant centres outside this region. On the whole, it appears to be less similar in structure to the evolved SVs 1 (e.g. Figures $1 \mathrm{a}$ and b) than are the evolved svs 1 to each other and to FTNM 1 . This is confirmed by calculating the pattern correlations between LV 1 and the evolved SVs 1 and FTNM 1 (not shown).

We now turn to the amplification factors of SVs, LVs and FTNMs. In the SF norm, Figure 2a shows the amplification factors between 20 and 26 April for FTNM 1, LV 1, EN SV 1, KE SV 1 and SF SV 1 in the inviscid case. Figure $2 \mathrm{~b}$ gives the corresponding singular values for SVs 1 in each of the three norms in the inviscid case. These singular values are also the amplification factors of SVs 1 in the respective norms. Also shown in Figure 2a are the mean and mean \pm standard deviation of 100 amplification factors of 100 initial randomly generated error fields in the SF norm in thick solid and thick 
dashed lines respectively. These random error fields, which are generated on 20 April, are chosen from a Gaussian distribution in which the magnitudes of the streamfunction spectral coefficients are proportional to $(2 l+1)^{-1}$; that is, $\left|\psi_{m l}\right| \sim \frac{1}{2 l+1}$. Here $m$ is zonal wave number, $l$ is total wave number and $\psi_{m l}$ are the streamfunction spectral coefficients. The corresponding kinetic energy spectra are then consistent with typical estimates of 2-day forecast errors.

We note that the amplification factors of FTNMs are just the magnitudes of the corresponding eigenvalues. The amplification factors of LVs 1 from 20 April to successive days have been calculated from the local Lyapunov exponents during these periods. The results show that FTNM 1 and LV 1 have very similar amplification factors in late April although, as we have noted, there are significant structural differences between FTNM 1 and LV 1 during this period. We also note from Figure 2a that FTNM 1 and LV 1 have similar, but slightly larger, amplification factors than the mean amplification factor of the 100 initially random errors in the SF norm. Also in the SF norm, the amplification factors of KE SV 1 and SF SV 1 are much larger than for LV 1 and FTNM 1 after a few days. After about three days the amplification factors of KE SV 1 and SF SV 1 are about an order of magnitude or more larger than the mean amplification factor of 100 initially random errors. However, in the SF norm, the amplification factors of EN SV 1 are lower than the mean amplification factor of 100 initially random errors in either the inviscid or viscous case. As expected, in both the inviscid and viscous cases, the amplification factors of SVs 1 in their respective norms are much larger than those of FTNM 1 and LV 1 as shown in Figure 2b. 

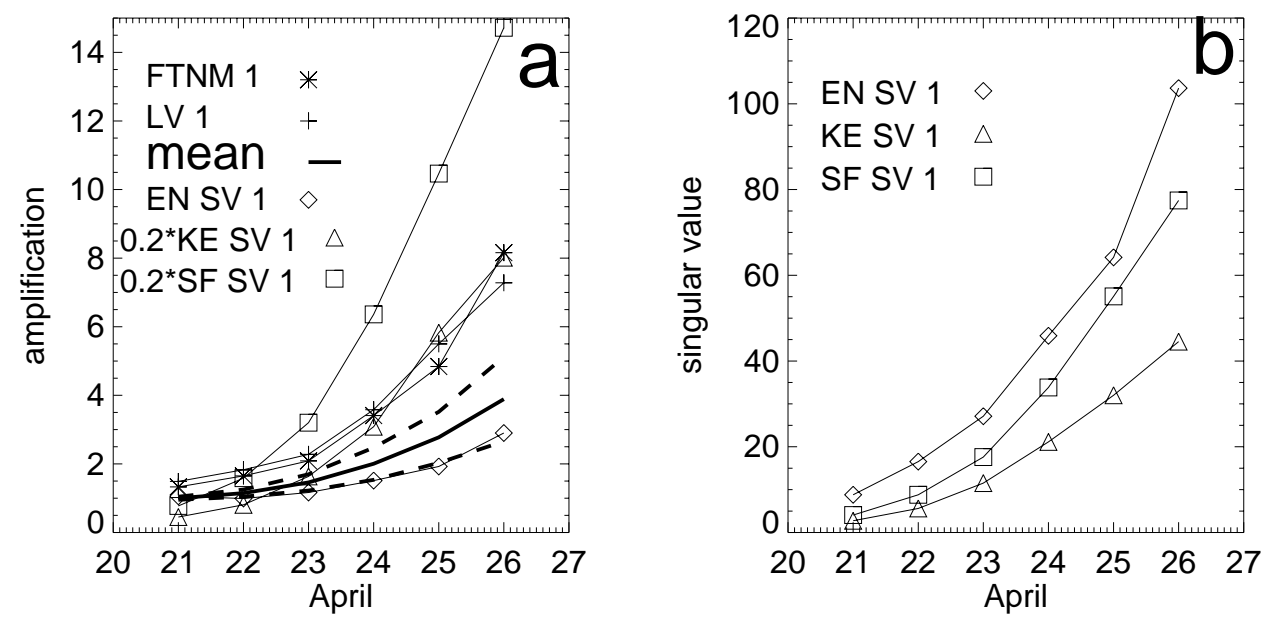

Figure 2: The amplification factors of dominant dynamical vectors in the inviscid case during periods from 20 to 21, 22, .., 26 April 1989. Shown are (a) amplification factors of FTNM 1 and LV 1, amplification factors in SF norm of EN SV 1, KE SV 1 (scaled by 0.2 ), SF SV 1 (scaled by 0.2 ) and the mean of 100 evolved errors (thick solid line) and mean \pm standard deviation of 100 evolved errors (thick dashed lines) in the inviscid case. Also shown in (b) are singular values of EN SV 1, KE SV 1, SF SV 1 in the inviscid case. 


\section{Statistics of Error Growth}

In this section, we determine the statistics of pattern correlations (calculated over the Southern Hemisphere) between 100 evolved, initially random, error fields and SVs, LVs and FTNMs. The initial random error fields are generated on 20 April as described in Section 3. The error fields are evolved in the tangent linear model out to 26 April. The mean (solid) and mean \pm standard deviation (dashed) of the pattern correlations between each of the 100 error fields and LVs are depicted in Figure 3a; we show these quantities for the largest correlations taken over the first five LVs and for both the inviscid (thin lines) and viscous (thick lines) cases.

We note that in Figure 3a, the mean and mean \pm standard deviations increase monotonically with time. There is little difference between the viscous and inviscid results in Figure 3a. There are generally higher correlations with LV 1 than LVs 2 to 5 (not shown), this is a reflection of the tendency for arbitrary initial disturbances to turn towards the leading Lyapunov vector. Figure 3a also shows (in the viscous case) the mean of the largest pattern correlations, taken over the five fastest growing FTNMs, with the 100 evolved error fields (thick dot-dashed line). We note that this mean is significantly higher (by one standard deviation, in fact) than for the mean involving the LVs.

Similarly, the mean of the pattern correlations with FTNM 1 is considerably higher than the mean with LV 1 except on 24 April when they are the 

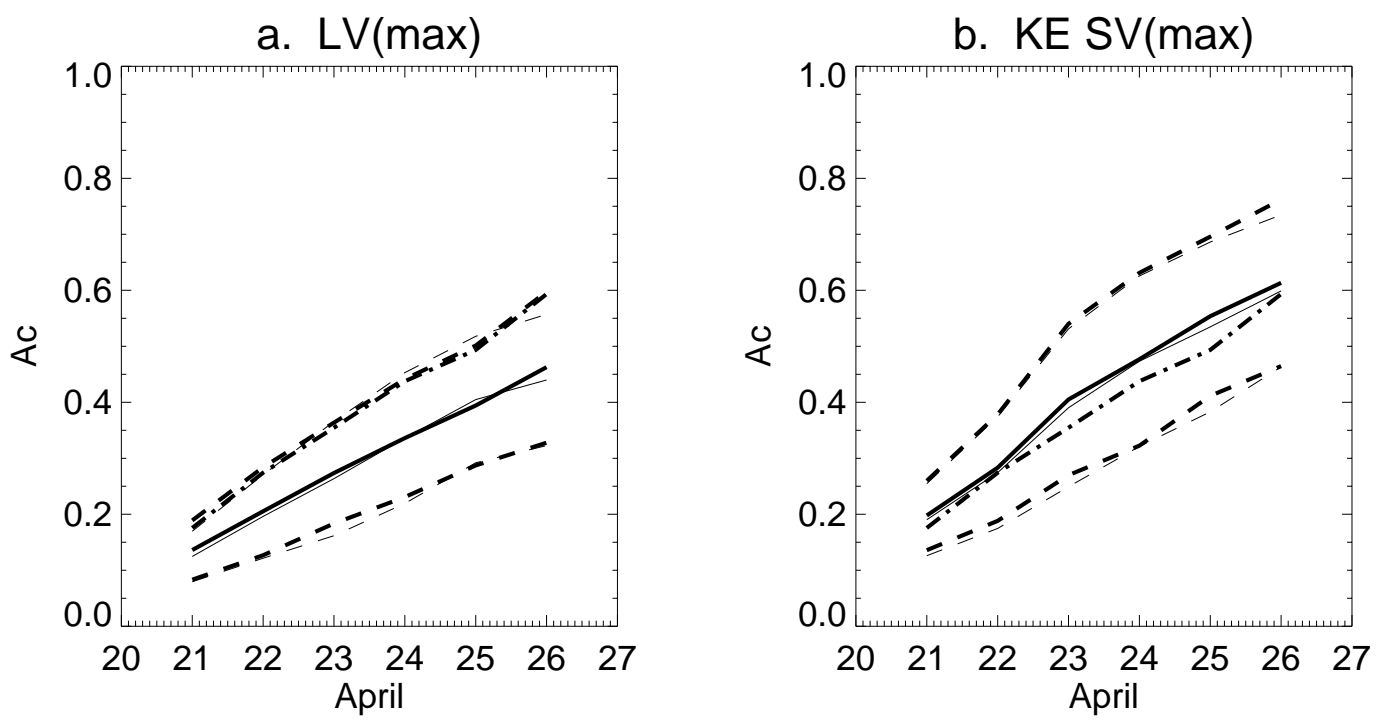

Figure 3: Pattern correlations $\left(A_{c}\right)$ between the dominant LVs, KE SVs and the 100 evolved random errors in both inviscid and viscous cases. Shown are (a) the mean (solid) and mean \pm the standard deviation (dashed) of the largest correlations taken over the five fastest growing LVs (viscous - thick, inviscid - thin) during the periods from 20 to 21, 22, .., 26 April 1989; also shown is the mean (thick dot-dashed) of the largest correlations taken over the five fastest growing FTNMs in the viscous case; (b) As in (a) but for KE SVs replacing LVs. 
same (not shown). In fact, on 24 April FTnMs 1 and 2 change role, with a larger mean pattern correlation between the 100 error fields and FTNM 2 than with FTNM 1 (not shown). These results indicate that initially random error fields that have only seen the tangent linear operator for a relatively short period of several days are better represented on average, in terms of dominant FTNMs for this same time interval, than by dynamical structures that have evolved from $t_{0} \rightarrow-\infty$ (dominant Lyapunov vectors).

Next, we present the statistics of pattern correlations between the 100 evolved error fields and SVs in the KE, EN and SF norms. Figures 3b depicts the statistics for the largest pattern correlations taken over the five fastest growing SVs in the KE norm and for both the viscous and inviscid cases. Again there is, in general, little difference in the results with and without viscosity, the statistics of pattern correlations increase monotonically with increasing time interval. We note that the mean pattern correlations with SVs in the KE norm and with FTNMs are, in general, very similar to each other. We have also found that mean pattern correlations with SVs in the SF norm are very similar to those in the KE norm and slightly larger than those with SVs in the EN norm. These mean pattern correlations are also larger than those with LVs shown in Figure 3a.

From the results presented in this section we can conclude that, after a few days, evolved, initially random, errors take up structures that are most similar to dominant SVs in the KE or SF norms or the dominant FTNMs. However, we know that the dominant SVs in SF and KE norms grow much too rapidly for the error fields to follow their time evolution. The amplification 
factors of the error fields are more closely similar to those of the dominant FTNMs and LVs.

\section{Conclusions}

In this paper, we have discussed and used iterative methods for producing SVs and LVs efficiently. We have compared the structures and evolutions of SVs and FTNMs with LVs growing on observed space-time basic states within a barotropic tangent linear model. The time-dependent basic states have been obtained by linear interpolation of observed daily 300-mb flow fields. We have focussed on synoptic situations in the Southern Hemisphere when blocks formed, amplified and decayed in the regions of Australia-New Zealand or in the central Pacific in April 1989. The growth of ensembles of 100 random initial errors have been analysed during this period of blocking.

The initial svs are found to depend sensitively on the specified norm. However, after a few days, the structures of the leading evolved svs in the KE, EN and SF norms, are in general quite similar and also similar to some of the dominant FTNMs that are norm independent. For optimisation times of six days or less, the evolved SVs and FTNMs are, in general, significantly different from the dominant LVs on the same day. Nevertheless, amplification factors of the first FTNMs and first LVs are very similar, and also similar to, but slightly larger than, the mean amplification factor of 100 initially random errors in the SF norm, while the amplification factors in the SF norm of KE SVs 1 and 
SF SV 1 are much higher. For longer optimisation times, the first SVs and the first FTNM increasingly turn towards the leading LV with convergence achieved within a month. We have examined the structural organisations of 100 initially random errors and compared their patterns with SVs, LVs and FTNMS.

\section{References}

[1] G. Benetin, L. Galgani, A. Giorgilli, and J.-M. Strelcyn. Lyapunov characteristic exponents for smooth dynamical systems and for Hamiltonian systems: a method for computing all of them. Meccanica, 15:9-30, 1980. C1507

[2] J.S. Frederiksen. Singular vectors, finite-time normal modes and error growth during blocking. J. Atmos. Sci., 57:312-333, 2000. C1503, C1505, C1508, C1509

[3] D.L. Harrar II and M.R. Osborne. Composite Arnoldi-Newton methods for large nonsymmetric eigenvalue problems. In B.J. Noye, M.D. Teubner and A.W. Gill, editors, Computational Techniques and Applications: CTAC97, pages 273-280, Singapore 1998. World Sci. Pub. C1505 
[4] F. Molteni, R. Buizza, T. Palmer, and T. Petroliagis. The ECMWF ensemble prediction system: methodology and validation. Quart. J. Roy. Meteor. Soc., 122:73-119, 1996. C1503, C1505, C1505

[5] Z. Toth and E. Kalnay. Ensemble forecasting at NCEP and the breeding method. Mon. Wea. Rev., 125:3297-3319, 1997. C1503, C1508

[6] M. Wei and J.S. Frederiksen. Applications of iterative eigensolvers to error growth during blocking in the southern hemisphere. In B.J. Noye, M.D. Teubner, and A.W. Gill, editors, Computational Techniques and Applications: CTAC97, pages 727-734, Singapore 1998. World Sci. Pub. C1505

[7] M. Wei, J.S. Frederiksen, and S. Kepert. Filtering methods for finite-time normal modes and atmospheric error growth. ANZIAM J., 42, 2000. [Online] http://anziamj . austms.org.au/V42/CTAC99/Wei1 C1504, C1504, C1506 